\title{
Clinical Efficacy and Safety of Bevacizumab, Apatinib, and Recombinant Human Endothelial Inhibitor in the Treatment of Advanced Gastric Cancer
}

\author{
Liang Wang, ${ }^{1}$ Wei Li, ${ }^{1}$ Ya-Gang Liu, ${ }^{1}$ Cui Zhang, ${ }^{1}$ Wei-Na Gao, ${ }^{2}$ and Li-Fei Gao ${ }^{3}{ }^{3}$ \\ ${ }^{1}$ The Second Department of General Surgery, Cangzhou Central Hospital, Cangzhou 061000, Hebei, China \\ ${ }^{2}$ The Fourth Department of Endocrinology, Cangzhou Central Hospital, Cangzhou 061000, Hebei, China \\ ${ }^{3}$ The Third Department of General Surgery, Cangzhou Central Hospital, Cangzhou 061000, Hebei, China \\ Correspondence should be addressed to Li-Fei Gao; gaolumu3859@163.com
}

Received 29 December 2021; Revised 14 January 2022; Accepted 25 January 2022; Published 24 February 2022

Academic Editor: Wei long Zhong

Copyright (c) 2022 Liang Wang et al. This is an open access article distributed under the Creative Commons Attribution License, which permits unrestricted use, distribution, and reproduction in any medium, provided the original work is properly cited.

Objective. To investigate the clinical efficacy and safety of bevacizumab, apatinib, and recombinant human endothelial inhibitor in the treatment of advanced gastric cancer. Methods. The medical data of 204 patients with a medium to advanced gastric cancer assessed for eligibility treated in our hospital from February 2019 to April 2020 were retrospectively analyzed. The eligible patients were assigned at a ratio of $1: 1: 1: 1$ to either the control group (chemotherapy), study group I (bevacizumab combined with chemotherapy), study group II (apatinib combined with chemotherapy), or study group III (recombinant human endothelial inhibitor combined with chemotherapy) according to different treatment methods. The treatment efficacy, drug toxicity, quality of life, and serum tumor marker levels before and after treatment were compared among the four groups. Results. Regarding the treatment effects, the effective rate of study group II (68.63\%) was significantly higher than that of the control group (33.33\%), study group I $(58.82 \%)$, and study group III $(49.02 \%)(P<0.05)$. The four groups showed similar safety and tolerability profiles $(P>0.05)$. The treatment in study group II led to a significantly higher physiological function score vs. the other three groups, but the scores of other items were not significantly different. Significant reduction was observed in the serum tumor markers after treatment in the four groups $(P<0.05)$, but treatment in study group II led to a significantly greater reduction than the other three groups $(P<0.05)$. Conclusion. The addition of apatinib, bevacizumab, and recombinant human endothelial inhibitor injection to chemotherapy for the treatment of medium to advanced gastric cancer can significantly improve the clinical treatment efficacy, among which the use of apatinib combined with chemotherapy achieves the best results, which is worthy of clinical promotion.

\section{Introduction}

Gastric cancer is a common gastrointestinal malignancy, with its prevalence and mortality rate ranking first among all malignancies in China. Its early clinical manifestations are insidious and nonspecific, which result in the progression of most cases to the advanced stage at the time of diagnosis, at which patients are usually inoperable [1]. For patients with advanced gastric cancer, disease control can only be achieved by chemotherapy. However, most patients are poorly tolerated under chemotherapy, experiencing toxic side effects, unfavorable therapeutic efficacy, and a high risk of deterioration [2]. Moreover, the survival benefits of patients with gastric cancer are modest, with the survival of advanced gastric cancer of about 4 months in the past progressing to 10 months to date. Remarkable efficiency has been achieved by targeted therapy in disease control of tumors. Bevacizumab is a new molecularly targeted antitumor drug in clinical practice, with promising antitumor effects as evidenced by current research reports and data [3-5]. Apatinib is a targeted drug that inhibits the multiplication of venous vascular endothelial cells to suppress the migration of endothelial cells [6-8]. The new recombinant human vascular endothelial inhibitor (Endostar) is a multitarget vascular endothelial inhibitor developed independently in China, which has obtained significant efficacy in 
the treatment of lung cancer. However, few scholars have been able to draw any systematic comparative research on the efficacy of these three drugs. Accordingly, this study included 204 patients with medium and advanced gastric cancer treated in our hospital from February 2019 to April 2020 as research subjects to evaluate the clinical efficacy and safety of bevacizumab, apatinib, and recombinant human endothelial inhibitor in treating advanced gastric cancer.

\section{Materials and Methods}

2.1. General Data. 204 patients with a medium to advanced gastric cancer treated in our hospital from February 2019 to April 2020 were identified as research subjects and were assigned to either control group (chemotherapy), study group I (bevacizumab combined with chemotherapy), study group II (apatinib combined with chemotherapy), or study group III (recombinant human endothelial inhibitor combined with chemotherapy) according to different treatment methods, with 51 cases in each group. This study was approved by the ethics committee of our hospital. The inclusion criteria for all study subjects were as follows: patients who were diagnosed with gastric cancer confirmed by pathological and cytological tests, patients aged between 30 and 80 years, and patients with an expected survival of more than 3 months. Exclusion criteria: patients with serious injurious diseases of the heart, liver, kidney, and other important organs; patients with psychiatric diseases; and patients in lactation or pregnancy [9].

\subsection{Methods}

2.2.1. Control Group. Oxaliplatin combined with 5-fluorouracil chemotherapy regimen: on day $1,90 \mathrm{mg} / \mathrm{m}^{2}$ of oxaliplatin (Hangzhou Zhongmei Huadong Pharmaceutical Co., Ltd., National Drug Administration H20113457, specification: $50 \mathrm{mg}$ ) was administered by intravenous drip within 3 hours. On days $1-5,500 \mathrm{mg} / \mathrm{m}^{2}$ of 5 -fluorouracil (Tianjin Jinyao Amino Acid Co., Ltd., National Drug Administration H12020959, specification: $250 \mathrm{mg}$ ) was administered intravenously after the drip. One cycle of chemotherapy spanned 21 days.

2.2.2. Study Group I. Bevacizumab was administered on the basis of the treatment in the control group. Bevacizumab (Roche, Switzerland) was administered at a dose of $7.5 \mathrm{mg} / \mathrm{kg}$ by intravenous infusion on day 1 , with a cycle of 21 days. The first administration of bevacizumab was performed 2 hours after chemotherapy, and the infusion was monitored for adverse reactions. The following administration of bevacizumab was conducted after chemotherapy. The efficacy of bevacizumab in combination with chemotherapy was evaluated once every cycle, and the treatment was continued in patients with effective cancer control until substantial remission of disease, intolerability, withdrawal of consent, or death.

2.2.3. Study Group II. Apatinib mesylate tablets were administered on the basis of the treatment in the control group.
Apatinib mesylate tablets (Jiangsu Hengrui Pharmaceutical Co., Ltd., State Drug Administration H20140103, specification: $0.25 \mathrm{~g} /$ tablet) were administered once daily half an hour after meals at an initial dose of $850 \mathrm{mg}$. Adverse reactions were monitored closely during treatment, and the treatment regimen was adjusted accordingly to allow for treatment tolerance, with a minimum adjusted dose of not less than $500 \mathrm{mg}$ once a day. The treatment was continued until documented disease progression or the development of intolerable adverse effects.

2.2.4. Study Group III. Recombinant human vascular endothelial inhibitor (Endostar) was administered on the basis of the treatment in the control group. Recombinant human vascular endothelial inhibitor (Endostar) (Shandong Xiansheng Medetzin Biopharmaceutical Co., Ltd., State Drug Administration S20050088, specification: $3 \mathrm{~mL}: 15 \mathrm{mg}$ each) was diluted with $0.9 \%$ sodium chloride injection at volume ratio of $1: 30$ and administered by intravenous drip for 3-4 hours, with an interval of 7 days after 14 days of administration.

The four groups of patients were treated for two cycles of treatment with one course of 21 days. After 42 days of treatment, a comprehensive examination was performed in all four groups. During chemotherapy, patients were closely monitored for bleeding and changes in liver and kidney function and white blood cell counts.

\subsection{Observation Indexes and Efficacy Criteria}

(i) Efficacy evaluation criteria: if the lesion disappears for more than 4 weeks after treatment, the efficacy is considered markedly effective. If the product of the maximum tumor diameter decreases by half or more after treatment and is maintained for more than 4 weeks, the efficacy is considered effective. If the product of the maximum tumor diameter decreases by less than $50 \%$ or increases by less than $25 \%$ after treatment, the efficacy is considered ineffective. If new lesions appear after treatment or the product of the maximum tumor diameter increases by more than $25 \%$, the efficacy is considered deteriorated. Total efficacy $=$ (markedly effective + effective)/cases* $100 \%$.

(ii) The incidence of toxic and side effects was monitored in four groups of patients. According to the National Cancer Institute Common Terminology Criteria for Adverse Events v3.0 (NCI-CTCAEv3.0) [10], the adverse reactions in this study were classified as grades I-IV. The incidence of adverse reactions in patients was also counted, which mainly included thrombocytopenia, cardiovascular toxicity, leukopenia, hemoglobin reduction, abnormal liver and kidney function, and nausea and vomiting.

(iii) The MOS 36-item short-form health survey (SF-36) [11] was used for quality of life measurement. The score of each item was counted and calculated into standard scores, standard scores $=$ (original score- 
lowest possible score)/the difference value of the highest score and the lowest score $\times 100 \%$; the sum of the standard scores of each dimension is the total score of SF-36, and higher score indicates better health or functional status.

(iv) The levels of glycoantigen 199 (CA199) and serum carcinoembryonic antigen (CEA) were determined in all groups before and after treatment using the electrochemiluminescence immunoassay [11].

2.4. Statistical Analyses. SPSS 26.0 was applied for statistical analysis. The count data were expressed as rates and analyzed using the chi-square test. The measurement data were expressed as mean \pm standard deviation $(\bar{x} \pm s)$, with the $t$-test for the comparison of the means between two groups and one-way ANOVA for the comparison of the means between multiple groups. The differences were statistically significant at $P<0.05$.

\section{Results}

3.1. Comparison of General Data of Four Groups of Patients. There was no significant difference in the general data of the four groups of patients $(P>0.05$, Table 1$)$.

3.2. Comparison of Treatment Efficacy. The total efficacy of patients was $33.33 \%$ in the control group, $58.82 \%$ in the study I group, $68.63 \%$ in the study II group, and $49.02 \%$ in the study III group $(P<0.05)$ (Table 2$)$.

3.3. Comparison of the Incidence of Adverse Reactions. No statistically significant differences were found in the incidence of thrombocytopenia, cardiovascular toxicity, leukopenia, and abnormal liver and kidney functions among the four groups $(P>0.05)$. The four groups had no serious adverse reactions (Table 3 ).

3.4. Comparison of Quality of Life Scores. The study groups outperformed the control group in terms of quality of life $(P<0.05)$ (Table 4).

3.5. Comparison of Serum Tumor Marker Levels. The four groups showed no significant differences in the serum tumor marker levels before treatment $(P>0.05)$. After treatment, the levels of CEA and CA199 were significantly decreased in the four groups $(P<0.05)$, in which the three study groups obtained significantly lower results than the control group $(P<0.05)$, and the study group II showed remarkably lower outcomes than the study groups I and III $(P<0.05)$ (Table 5).

\section{Discussion}

Gastric cancer is a common malignancy with insidious and variable symptoms in the early stage. The disease has mostly progressed to the advanced stage by the time of diagnosis. Patients with advanced gastric cancer are mostly inoperable, with great difficulty in treatment, poor prognosis, and high mortality rates. Statistically, more than $50 \%$ of patients undergoing surgery are associated with in situ recurrence or distant metastasis, which undermines patients' physical and mental health and compromises their quality of life. Currently, chemotherapy is the mainstay for treating advanced gastric cancer, which underscores the significance of the scientific application of chemotherapy drugs to enhance the treatment efficiency and avoid adverse reactions. In tumor growth and metastasis, neovascularization is a major influencing factor, so the exploration of therapeutic methods targeting antitumor angiogenesis remains a key issue to be addressed. Previous studies have revealed that angiogenesis inhibitors could coordinate or even superimpose the effects of cytotoxic agents or radiation therapy $[5,6]$.

Bevacizumab is a monoclonal antibody that inhibits the vascular endothelial growth factor and is mainly used for metastatic cancer treatment, with the mechanism to inhibit the mitosis of tumor vascular endothelial cells and angiogenesis by blocking or reducing the binding of the vascular endothelial growth factor to its corresponding receptor. In addition, it can lead to insufficient blood oxygenation of growing and multiplying tumor cells by disrupting tumor vasculature to facilitate the contact of chemotherapeutic drugs with tumors $[12,13]$. Apatinib is a derivative compound of PTK787 and is the first generation of smallmolecule tyrosine kinase inhibitor targeting the angiogenic signaling pathway developed clinically in China and the latest oral targeted therapeutic agent against tumor angiogenesis. It effectively binds ligands such as vascular endothelial growth factor VEG-FR-2 and blocks the signaling after VEGF [14] binding, thereby inhibiting tumor neovascularization [15]. Recombinant human vascular endothelial inhibitor is an endogenous factor that inhibits neovascularization by binding and phagocytosing proteins of endothelial cells to block their signaling pathways and to mediate the expression profile of endothelial cells. The efficacy of recombinant human vascular endothelial inhibitor on tumors is mediocre during single-use but can be potentiated in a joint administration. Cisplatin features an extremely strong peritoneal nodal penetration, and docetaxel and tegafur capsules can disrupt the microvascular meshwork, which, together with the inhibitory and modulatory effects of recombinant human endothelial inhibitor, effectively boosts the therapeutic effect. Prior studies have confirmed the significant clinical efficacy of bevacizumab, apatinib, and recombinant human endothelial inhibitor in combination with chemotherapy in the treatment of patients with a medium to advanced gastric cancer $[16,17]$.

In this study, the higher efficacy in all three study groups versus the control group indicated that the combination of chemotherapy with apatinib, bevacizumab, and recombinant human endothelial inhibitor potentiated the effectiveness of chemotherapy drugs in inhibiting tumor growth. In terms of treatment efficiency, the study group II outperformed the study group I, followed by the study group III, indicating that the efficacy of apatinib combined with chemotherapy was better than that of bevacizumab and recombinant human endothelial inhibitor combined with 
TABLE 1: Comparison of general data of the four groups of patients $(\bar{x} \pm s)$.

\begin{tabular}{lcccccc}
\hline Groups & $\begin{array}{c}\text { Gender (male/female) } \\
\text { (case) }\end{array}$ & $\begin{array}{c}\text { Mean age } \\
\text { (year) }\end{array}$ & $\begin{array}{c}\text { Mean course of disease } \\
\text { (months) }\end{array}$ & \multicolumn{3}{c}{ Stage (case) } \\
IIIa & IIIb & IIIc & IV \\
\hline Control group & $35 / 16$ & $48.62 \pm 6.87$ & $4.61 \pm 1.53$ & 11 & 20 & 11 \\
Study I group & $32 / 19$ & $51.04 \pm 7.98$ & $5.02 \pm 2.31$ & 10 & 22 & 10 \\
Study II group & $36 / 15$ & $50.04 \pm 5.34$ & $4.87 \pm 2.75$ & 11 & 21 & 9 \\
Study III group & $34 / 17$ & $49.77 \pm 8.23$ & $4.91 \pm 1.83$ & 12 & 19 & 10 \\
$X^{2} / F$ & 0.482 & 0.975 & 0.331 & 0.232 & 0.408 & 0.249 \\
$P$ & 0.546 & 0.406 & 0.803 & 0.975 & 0.939 & 0.969 \\
\hline
\end{tabular}

TABLE 2: Comparison of treatment efficacy of patients in the four groups $(n(\%))$.

\begin{tabular}{|c|c|c|c|c|c|c|}
\hline Groups & $n$ & Markedly effective & Effective & Ineffective & Deteriorated & Total efficacy \\
\hline Control group & 51 & 4 & 13 & 27 & 7 & $33.33 \%$ \\
\hline Study group I & 51 & 8 & 22 & 18 & 2 & $58.82 \%^{\mathrm{a}}$ \\
\hline Study group II & 51 & 10 & 25 & 15 & 1 & $68.63 \%^{\mathrm{a}}$ \\
\hline Study group III & 51 & 6 & 19 & 23 & 3 & $49.02 \%^{\mathrm{a}}$ \\
\hline$X^{2}$ & & & & & & 15.259 \\
\hline$P$ & & & & & & 0.002 \\
\hline
\end{tabular}

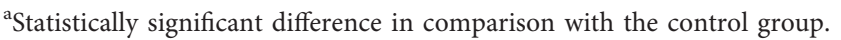

TABLE 3: Comparison of the incidence of adverse reactions among the four groups of patients ( $n(\%))$.

\begin{tabular}{|c|c|c|c|c|c|c|c|c|c|c|c|c|c|}
\hline \multirow[t]{2}{*}{ Groups } & \multirow[t]{2}{*}{$n$} & \multicolumn{2}{|c|}{ Thrombocytopenia } & \multicolumn{2}{|c|}{$\begin{array}{l}\text { Cardiovascular } \\
\text { toxicity }\end{array}$} & \multicolumn{2}{|c|}{ Leukopenia } & \multicolumn{2}{|c|}{$\begin{array}{l}\text { Hemoglobin } \\
\text { reduction }\end{array}$} & \multicolumn{2}{|c|}{$\begin{array}{l}\text { Abnormal } \\
\text { liver and } \\
\text { kidney } \\
\text { functions }\end{array}$} & \multicolumn{2}{|c|}{$\begin{array}{c}\text { Nausea and } \\
\text { vomiting }\end{array}$} \\
\hline & & I-II & III-IV & I-II & III-IV & I-II & III-IV & I-II & III-IV & I-II & III-IV & I-II & III-IV \\
\hline Control group & 51 & 20 & 17 & 15 & 14 & 19 & 14 & 18 & 10 & 18 & 12 & 22 & 11 \\
\hline Study group I & 51 & 21 & 15 & 15 & 13 & 21 & 17 & 15 & 11 & 19 & 17 & 25 & 13 \\
\hline Study group II & 51 & 20 & 14 & 14 & 11 & 17 & 17 & 14 & 13 & 18 & 16 & 23 & 12 \\
\hline Study group III & 51 & 23 & 16 & 16 & 16 & 19 & 18 & 17 & 16 & 20 & 16 & 26 & 14 \\
\hline
\end{tabular}

TABLE 4: Comparison of quality of life scores among patients in the four groups ( $\bar{x} \pm s$, points).

\begin{tabular}{lccccccc}
\hline Groups & Physical functioning & Bodily pain & Social functioning & Role emotional & Mental health & Vitality & General health \\
\hline Control group & $69.31 \pm 5.76$ & $54.41 \pm 4.35$ & $49.87 \pm 4.12$ & $50.11 \pm 5.64$ & $61.87 \pm 6.85$ & $59.32 \pm 7.13$ & $65.97 \pm 4.63$ \\
Study group I & $79.11 \pm 5.48$ & $55.28 \pm 5.79$ & $53.46 \pm 6.75$ & $51.34 \pm 4.97$ & $70.55 \pm 5.49$ & $61.55 \pm 6.82$ & $78.11 \pm 6.31$ \\
Study group II & $83.57 \pm 6.49$ & $56.34 \pm 5.91$ & $54.51 \pm 5.74$ & $54.73 \pm 5.17$ & $74.66 \pm 6.53$ & $66.33 \pm 7.28$ & $78.94 \pm 7.03$ \\
Study group III & $80.42 \pm 6.34$ & $55.30 \pm 3.36$ & $53.36 \pm 4.93$ & $52.44 \pm 4.23$ & $74.32 \pm 6.78$ & $65.09 \pm 4.87$ & $77.57 \pm 4.82$ \\
$F$ & & & & & & 61.95 \\
$P$ & & & & & & \\
\hline
\end{tabular}

TABLE 5: Comparison of serum tumor marker levels before and after treatment in the four groups $(\bar{x} \pm s, \mathrm{mg} / \mathrm{L})$.

\begin{tabular}{|c|c|c|c|c|c|}
\hline \multirow{2}{*}{ Groups } & \multirow{2}{*}{$n$} & \multicolumn{2}{|c|}{ CEA } & \multicolumn{2}{|c|}{ CA199 } \\
\hline & & Before treatment & After treatment & Before treatment & After treatment \\
\hline Control group & 51 & $67.51 \pm 13.48$ & $33.14 \pm 5.53^{*}$ & $436.75 \pm 52.64$ & $246.34 \pm 30.01^{*}$ \\
\hline Study group I & 51 & $68.31 \pm 12.37$ & $18.47 \pm 5.34^{* \mathrm{a}}$ & $431.79 \pm 48.99$ & $185.37 \pm 26.51^{* a}$ \\
\hline Study group II & 51 & $68.49 \pm 11.64$ & $10.36 \pm 6.13^{* \mathrm{a}}$ & $435.17 \pm 49.61$ & $163.74 \pm 27.16^{* \mathrm{a}}$ \\
\hline Study group III & 51 & $67.99 \pm 12.08$ & $17.97 \pm 5.83^{* \mathrm{a}}$ & $434.64 \pm 50.36$ & $190.34 \pm 29.44^{* \mathrm{a}}$ \\
\hline$F$ & & 88.233 & 141.582 & 73.213 & 78.839 \\
\hline$P$ & & 0.021 & $<0.001$ & 0.011 & $<0.001$ \\
\hline
\end{tabular}

${ }^{*} P<0.05$, in the comparison with pretreatment. ${ }^{a} P<0.05$, in the comparison between the study groups and the control group after treatment.

chemotherapy. Assumedly, apatinib highly selectively competes with intracellular vascular endothelial growth factor receptor 2 , and binds to it, thereby inhibiting tumor angiogenesis and reducing tumor blood supply, oxygen supply, and other nutrients supply to tumor growth [17-19]. The absence of statistically significant differences in the 
incidence of adverse reactions among the four groups indicates the treatment regimens used in the study groups did not significantly increase the toxicity of the drugs with a high safety profile $[20,21]$. The three study groups presented superior improvement in the quality of life to that of the control group; additionally, the levels of CEA and CA199 were markedly reduced in the four groups after treatment, with better results in the study groups, indicating that the combined treatment regimens employed in this study yielded more favorable outcomes than the stand-alone chemotherapy. Furthermore, the study group II showed remarkably lower levels of CEA and CA199 versus the study groups I and III.

\section{Conclusion}

The addition of apatinib, bevacizumab, and recombinant human endothelial inhibitor injection to chemotherapy for the treatment of medium to advanced gastric cancer can significantly improve the clinical treatment efficacy, among which the use of apatinib combined with chemotherapy achieves the best results, which is worthy of clinical promotion.

\section{Data Availability}

The datasets used to support the findings of this study are available from the corresponding author upon request.

\section{Conflicts of Interest}

The authors declare that they have no conflicts of interest.

\section{References}

[1] Z. Song, Y. Wu, J. Yang, D. Yang, and X. Fang, "Progress in the treatment of advanced gastric cancer," Tumour Biology, Article ID 1010428317714626, 2017.

[2] R. Reddavid, S. Sofia, P. Chiaro et al., "Neoadjuvant chemotherapy for gastric cancer. Is it a must or a fake?" World Journal of Gastroenterology, vol. 24, no. 2, pp. 274-289, 2018.

[3] B. Hultman, U. Gunnarsson, P. Nygren, M. Sundbom, B. Glimelius, and H. Mahteme, "Prognostic factors in patients with loco-regionally advanced gastric cancer," World Journal of Surgical Oncology, vol. 15, no. 1, p. 172, 2017.

[4] L. Wang, W. Li, Y. Liu, C. Zhang, W. Gao, and L. Gao, "Clinical study on the safety, efficacy, and prognosis of molecular targeted drug therapy for advanced gastric cancer," American Journal of Tourism Research, vol. 13, no. 5, pp. 4704-4711, 2021.

[5] P. Gotwals, S. Cameron, D. Cipolletta et al., "Prospects for combining targeted and conventional cancer therapy with immunotherapy," Nature Reviews Cancer, vol. 17, no. 5, pp. 286-301, 2017.

[6] T. H. Patel and M. Cecchini, "Targeted therapies in advanced gastric cancer," Current Treatment Options in Oncology, vol. 21, no. 9, p. 70, 2020.

[7] J. Garcia, H. I. Hurwitz, A. B. Sandler et al., "Bevacizumab (Avastin) in cancer treatment: a review of 15 years of clinical experience and future outlook," Cancer Treatment Reviews, vol. 86, Article ID 102017, 2020.
[8] J. Xue, M. Astère, M.-X. Zhong, H. Lin, J. Shen, and Y.-X. Zhu, "Efficacy and safety of apatinib treatment for gastric cancer, hepatocellular carcinoma and non-small cell lung cancer: a meta-analysis," OncoTargets and Therapy, vol. 11, pp. 6119-6128, 2018.

[9] N. Charalampakis, P. Economopoulou, I. Kotsantis et al., "Medical management of gastric cancer: a 2017 update," Cancer Medicine, vol. 7, no. 1, pp. 123-133, 2018.

[10] A. R. Yusefi, K. Bagheri Lankarani, P. Bastani, M. Radinmanesh, and Z. Kavosi, "Risk factors for gastric cancer: a systematic review," Asian Pacific Journal of Cancer Prevention: Asian Pacific Journal of Cancer Prevention, vol. 19, no. 3, pp. 591-603, 2018.

[11] C. Röcken, "Molecular classification of gastric cancer," Expert Review of Molecular Diagnostics, vol. 17, no. 3, pp. 293-301, 2017.

[12] J. H. Kim, S. R. Park, M.-H. Ryu et al., "Phase II study of induction chemotherapy with docetaxel, capecitabine, and cisplatin plus bevacizumab for initially unresectable gastric cancer with invasion of adjacent organs or paraaortic lymph node metastasis," Cancer Research and Treatment, vol. 50, no. 2, pp. 518-529, 2018.

[13] Y. Lv, L. Song, L. Chang, X. Zhang, Y. Liu, and W. Liu, "Effect of bevacizumab combined with chemotherapy at different sequences in the gastric-cancer-bearing nude mice," Journal of Cancer Research and Therapeutics, vol. 14, no. Supplement, pp. s190-s196, 2018.

[14] B. Mawalla, X. Yuan, X. Luo, and P. L. Chalya, "Treatment outcome of anti-angiogenesis through VEGF-pathway in the management of gastric cancer: a systematic review of phase II and III clinical trials," BMC Research Notes, vol. 11, no. 1, p. 21, 2018.

[15] L. J. Scott, "Apatinib: a review in advanced gastric cancer and other advanced cancers,” Drugs, vol. 78, no. 7, pp. 747-758, 2018.

[16] L. Guo, B. Xu, D. Zhou et al., "Biophysical and biological characterization of PEGylated recombinant human endostatin," Clinical and Experimental Pharmacology and Physiology, vol. 46, no. 10, pp. 920-927, 2019.

[17] K. Li, M. Shi, and S. Qin, "Current status and study progress of recombinant human endostatin in cancer treatment," Oncology and Therapy, vol. 6, no. 1, pp. 21-43, 2018.

[18] W. H. Allum, E. C. Smyth, J. M. Blazeby et al., "Quality assurance of surgery in the randomized ST03 trial of perioperative chemotherapy in carcinoma of the stomach and gastro-oesophageal junction," British Journal of Surgery, vol. 106, no. 9, pp. 1204-1215, 2019.

[19] K. Uemura, H. Takahashi, K. Mino, T. Ota, and S. Shichi, "[Four cases of gastrointestinal perforation associated with bevacizumab]," Gan To Kagaku Ryoho, vol. 44, no. 12, pp. 1604-1606, 2017.

[20] E. Wöll, J. Thaler, F. Keil et al., "Oxaliplatin/irinotecan/ bevacizumab followed by docetaxel/bevacizumab in inoperable locally advanced or metastatic gastric cancer patients AGMT_GASTRIC-3," Anticancer Research, vol. 37, no. 10, pp. 5553-5558, 2017.

[21] J. Yao, L. Fan, C. Peng et al., "Clinical efficacy of endostar combined with chemotherapy in the treatment of peritoneal carcinomatosis in gastric cancer: results from a retrospective study," Oncotarget, vol. 8, no. 41, pp. 70788-70797, 2017. 